\title{
EL DISCURSO DEL PROFESOR Y DEL ALUMNO: ANÁLISIS DIDÁCTICO EN CLASES DE CIENCIAS
}

\author{
DE LONGHI, ANA LÍA \\ Facultad de Matemática Astronomía y Física. Ciudad Universitaria. Córdoba. Argentina. \\ E-mail: delonghi@mate.uncor.edu
}

\begin{abstract}
SUMMARY
This article is the first part of a research on interpreting students' and teachers' discourse in Science classes. Its aim is to contribute to the understanding of the construction of knowledge that emerges through verbal messages between teacher and student in interaction.

A four-level analisis is developed. Firstly the observable class features are described; secondly different categories of teacher and student interventions are presented.

At the third level, inferences on didactical aspects are drawn from the previous levels. At the fourth level the sequence of the subject-matter organization in studied for each curriculum item.

Finally the communication problem from a didactical point of view is discussed.
\end{abstract}

\section{INTRODUCCIÓN}

La historia de las investigaciones en didáctica de las ciencias revela la necesidad de integrar a su marco teórico elementos de psicología, pedagogía, epistemología y sociología. Actualmente, este cuerpo de conocimientos debe considerar necesariamente la renovación en la concepción epistemológica de las ciencias, los avances de la psicología cognitiva, los movimientos relacionados con el enfoque constructivista y el modelo didáctico investigativo, basados principalmente en las concepciones previas y en la ecología de la construcción de los saberes. En éste ámbito surge como línea de investigación el análisis de la estructura y dinámica de la comunicación en el aula (Astolfi y Develay, 1989). Centrados en la idea de que el conocimiento científico es una construcción social, producto del esfuerzo humano, más que un conocimiento objetivo, los constructivistas sostienen que el proceso de aprendizaje resulta de la interacción entre los esquemas mentales del que aprende y las características del contexto de aprendizaje (Driver,
1989); cobran valor las ideas previas, las estrategias cognitivas, metacognitivas y los propósitos e intereses de los alumnos; importa el sentido de cada situación de enseñanza y aprendizaje para cada individuo, grupo y momento. Se retoma la dimensión interaccionista del aprendizaje y se tiene en cuenta que la construcción del conocimiento depende de un proceso de negociación social (Edwards y Mercer, 1987). Dicha construcción deja de ser un hecho solamente individual y se rescata la complejidad de las relaciones que involucra (Mortimer, 1996).

Comprender dicha complejidad y analizar cómo circula un conocimiento en situaciones de clase, en el marco del proceso de comunicación en que éste participa, requiere la delimitación de aspectos teóricos específicos y la elaboración de una metodología particular. A pesar de que hay numerosas investigaciones en didáctica de las ciencias relacionadas con la realidad del aula, no conta- 
mos con demasiadas producciones que hagan referencia a las nociones teóricas que surgen del análisis de las intervenciones instruccionales (Thorley y Stofflett, 1996), a los problemas relacionados con la interpretación de dichas intervenciones (Klaassen y Lijnse, 1996) o a los procesos de comunicación interpersonales y grupales, como los contextos que posibilitan el cambio esperado en la enseñanza o en el aprendizaje de determinado tema.

El estudio que presentamos aquí está centrado en el análisis de la comunicación que se establece, a través del diálogo docente-alumnos, a partir de determinado objeto de conocimiento de una disciplina científica. El objetivo del mismo es conformar un esquema de análisis que permita identificar e interpretar las formas de intervención verbal que se originan en la enseñanza del contenido desde un marco teórico propio para la didáctica de las ciencias.

Este trabajo se enmarca en una concepción de la didáctica de las ciencias dedicada más al análisis e investigación de la multidimensionalidad del proceso de enseñanza y aprendizaje que a una finalidad puramente instrumental y prescriptiva. Para nosotros, la clase constituye una realidad singular y compleja, donde se produce un conjunto de relaciones que provocan la comunicación entre sus agentes y el conocimiento específico. Las interacciones y negociaciones entre docente y alumnos que en ella ocurren permiten la circulación de dicho conocimiento en el marco de contextos personales e institucionales cambiantes. Por ello sus principales componentes son: el docente, los alumnos, el objeto de conocimiento, los contextos (situacional, lingüístico y mental), sus relaciones y procesos (De Longhi, 1994). Por el carácter singular, asimétrico e intencional de cada clase cuestionamos la factibilidad de identificar formas tipo o patrones en dicha comunicación didáctica y preferimos buscar indicadores que permitan la interpretación a partir de un modelo didáctico específico para su análisis.

\section{LA COMUNICACIÓN DIDÁCTICA: UN MARCO TEÓRICO EN DESARROLLO}

Un emergente fundamental de la relación social de enseñar y aprender es el lenguaje. Desde los aportes sociolingüísticos, como los de Berstein (1977, 1988), Stubbs (1984) y Cazden (1988), se muestra que los códigos y los contextos de referencia del habla influyen en la construcción de significados tanto personales como sociales en el marco de la escuela.

Desde los años sesenta encontramos diferentes investigaciones que toman como objeto de estudio el diálogo en el aula (Bellack, 1966; Doyle, 1977) y formulaciones de categorías de preguntas y respuestas (Barnes, 1969; Sinclair y Couthard, 1975; Titone, 1986). Desde las posturas pedagógicas interestructurantes, como las de Not (1983) y Perret-Clermont (1984), se señala la necesidad de propiciar actividades que generen interacciones en el aula, donde el alumno pueda participar, se adecúe el nivel de ayuda a su nivel de competencia y se respeten sus tiempos de construcción. Así, la estructura de la tarea académica y el intercambio docente-alumno se alejaría de la marcada intención evaluativa que suele caracterizarla.

Vigotsky (1979), desde la psicología, fundamenta el lugar que ocupa la mediación que provoca el lenguaje en el aprendizaje. Muestra la necesidad de comprender la distancia que debe recorrer el niño entre lo que ya sabe y lo que puede llegar a aprender con ayuda de otro. Hoy ya no se discute la importancia de la construcción de significados propios por parte del alumno y del rol fundamental que ocupa el docente, por el andamiaje (Bruner, 1988) que puede proporcionar al proceso de aprendizaje de sus alumnos.

En la década de los años ochenta la preocupación principal de los investigadores en enseñanza de las ciencias estuvo ligada al análisis de los aprendizajes de los alumnos, a sus preconcepciones y a las de los docentes. Recién finalizada dicha década se comienza a relacionar el aprendizaje con el contexto social en que esta inmerso (Edwards y Mercer, 1987, 1988; Seeger, 1991) y con las expresiones verbales y argumentaciones que se presentan en las clases de ciencias (Llorens 1987, 1998; Candela 1991).

Desde diferentes perspectivas, pedagógica, psicológica o sociolingüística, se proponen análisis adecuados, aunque poco específicos respecto de lo que ocurre con la construcción del conocimiento científico y con escasas teorizaciones sobre las situaciones particulares en que éstos se generan; por ello creemos que es necesario conformar una explicación didáctica de tales hechos.

La comunicación didáctica es un caso particular de la comunicación humana, caracterizada por involucrar dos procesos, el de enseñanza y el de aprendizaje y por ser asimétrica, intencional e institucionalizada (De Longhi, 1995). Hablar de comunicación entre docente y alumnos implica analizar un microsistema social, el aula, dentro de la escuela, en un lugar y durante un período de tiempo, dedicados y organizados para facilitar y desarrollar este tipo de intercambio.

El análisis de dicho proceso, como un sistema (Bertalanffy, 1976; Pérez Gómez, 1985; Mayor Sánchez, 1983), garantiza la consideración de su complejidad, con todas las variables y relaciones que incluye, donde cada individuo es un subsistema que se comunica con otro y experimenta intercambios significativos. De acuerdo con Watzalawick (1981), la comunicación didáctica se presenta como un sistema abierto en el cual el individuo no sólo participa u origina una comunicación sino que se involucra, «entra en una comunicación».

Desde nuestra perspectiva a los cuatro elementos clásicos del modelo comunicacional (emisor, receptor, mensaje y contexto), en el diálogo del aula, con el fin de enseñar y aprender un conocimiento disciplinar, deben agregarse otros más específicos. En la figura 1 mostramos una representación de la dinámica de la relación 
INVESTIGACIÓN DIDÁCTICA

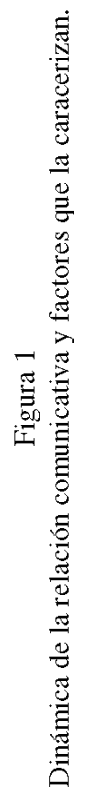


comunicativa docente-alumnos y los factores que la caracterizan.

Inicialmente debemos considerar que, si la metodología de enseñanza propuesta por un docente de ciencias permite la interestructuración del conocimiento, tanto él como sus alumnos se convierten en emisores y receptores a la vez y, acorde a su actuación y cognición, provocan una mediación en la comunicación de los mensajes. A medida que dicho mensaje circula, cada uno de ellos lo va resignificando a partir de las construcciones personales que realiza.

La relación entre dichos procesos, de enseñar y aprender, es complementaria más que causal (Fentermacher, 1986) y conlleva una asimetría de funciones entre docente y alumnos, porque es el enseñante quien desencadena y orienta la participación del alumno. Según Watzalawick (1981) la relación profesor-alumno es «asimétricamente contingente», ya que la posición del profesor es substancialmente y permanentemente predominante.

Las principales diferencias entre distintos docentes está dada por el tipo de relación que cada uno de ellos establece con el saber, que se manifiesta en su dominio de la materia y el significado que da al contenido propuesto en su enseñanza, la forma de situarse y de situar al alumno con relación a dicho saber, los marcos de referencia que sugiere o impone y los tiempos de construcción conceptual que otorga o niega. Esta asimetría tiene grados que pueden llegar a un punto extremo donde no ocurra comunicación en el aula. En la elaboración conjunta de un conocimiento, el docente regula el ritmo y tiempos de construcción, que no siempre contemplan las posibilidades del alumno, haciendo valer su autoridad tanto académica como funcional. Si bien el docente personifica el conocimiento realmente enseñado, sus fuentes previas de validación no siempre se originan en el conocimiento científico actualizado. Muchas veces la rutina de la docencia hace que tome sólo su experiencias previas como criterio de validez, tanto las referidas al contenido disciplinar como las relacionadas con el conocimiento pedagógico didáctico correspondiente a la enseñanza de determinada materia y al posible aprendizaje.

Son numerosas las investigaciones que, a través del estudio de las reglas básicas del discurso educacional, llegan a la conclusión de que el docente controla el discurso y actúa como árbitro de la validez de los conocimientos a través de un conjunto de reglas (explícitas o implícitas) que están presentes en el habla del aula. Dichas reglas no sólo aluden a la correspondencia entre significante y significado, sino que también regulan e interpretan la interacción que se establece. Como expresan Edwards y Mercer (1988, p. 144): «Es dentro del discurso maestro-alumno, en el cual se desarrolla la lección, donde se modelan, interpretan, destacan, limitan a lo periférico, reinterpretan, etc., todas las comprensiones que se crean.»

Por otro lado, el alumno participa de una actividad didáctica específica, transitando por diferentes niveles de comunicación. El primero es el intraindividual (Pérez Gómez,1985) e intrapsicológico, que permite revisar sus conocimientos y referentes. Posteriormente, por intercambio con otros, se pasa a un nivel interindividual, que provoca la circulación del mensaje que se explicita y comparte en el aula. Por último, los niveles anteriores interactúan con otro llamado situacional, fuertemente influido por las características de la institución en que ocurre y de los diseños curriculares que las rigen.

También debemos comprender que los conocimientos involucrados en los mensajes escolares se producen en un momento histórico-cultural determinado. El docente los trae al aula y recontextualiza, creando situaciones para su desarrollo. Dependerá de la ocurrencia de dichas situaciones de clase que el alumno logre «re-des-contextualizarlos» (Brousseau, 1996), es decir, construir significados y procesos personales.

Lo que se habla o calla, acepta o niega en el diálogo de una clase de ciencias está regulado, como expresábamos en un comienzo, por tres tipos de contextos: el situacional, el lingüístico (Stubbs, 1984) y el mental (Edwards y Mercer, 1987). El primero lo sitúa en un lugar, una cultura y una institución. El segundo es el que se genera por el propio discurso y se relaciona con los códigos de habla, el lenguaje de la disciplina, su lógica y la generada por la interacción en la clase. El tercero corresponde a los niveles interindividual e intraindividual y a las posibilidades de aprendizaje del grupo. Este último contexto mental representa las comprensiones generales que surgen entre las personas que se comunican, ya no como un hecho lingüístico, ni como producto de las circunstancias físicas, sino como ayuda a los participantes a dar sentido a lo que dicen (Edwards y Mercer, 1987). Hemos observado que, a medida que el grupo clase comparte más tiempo y comunicaciones en un ámbito disciplinar específico, se van acordando dichos contextos lingüísticos y mentales y se produce un intercambio más fluido. En estas condiciones es cuando se elaboran y transmiten significados, se comparten referentes y el docente actúa como regulador del diálogo, facilitando la interestructuración de contenidos, en un esfuerzo por compartir su contrucción.

El mensaje (verbal o gestual) presente en las aulas funciona como mediador entre las personas (subsistemas personales) que intervienen en la comunicación; se elabora y emite a través de códigos que representan significados dados por determinados significantes. En la escuela, algunos mensajes, referidos a contenidos específicos, ya se encuentran semántica y sintácticamente estructurados en los libros de texto, en el currículo y hasta en las guías de clase. La distancia entre éstos y los mensajes verbales que originan las diferentes actividades de la clase son fuente de problema en la comunicación didáctica. Muchas veces, a pesar de observar la participación de los alumnos en el diálogo, la lógica generada por la interacción verbal entre ellos y el docente desvirtúa la lógica del contenido académico propuesto. Es aquí donde cobra importancia el estilo docente, las situaciones que organiza y el uso de una sintaxis y semántica particular que transporta significados al 
alumno y lo conduce, a través de la interacción, a un determinado nivel de construcción del contenido. Es una relación de influencia que le da a los significados un carácter dinámico que en el lenguaje se manifiestan fluctuantes y muchas veces se definen por el uso de las palabras más que por sus referentes (Llorens et al., 1989).

Como expresa Titone (1981), los dos comunicantes en la escuela pueden actuar significativamente tan sólo si poseen «competencia comunicativa» suficientemente homogénea que, además de la uniformidad de códigos (aspectos formales), incluya convergencia sociocultural, cognitiva y dinámico-afectiva.

El carácter del contenido es otro de los elementos que influye en el proceso de comunicación, no sólo por la forma en que se hace explícito en el aula, sino también por su ubicación disciplinar, lógica particular, selección y organización curricular, grado de formalización, distancia respecto del conocimiento cotidiano y conocimientos previos que requiere. Así, un contenido más cercano a la experiencia del alumno favorece una dinámica de interacción en la clase y posibilita la participación del estudiante.

El diálogo puede centrarse en el contenido, o en la forma de conocerlo, por ejemplo, en un tema específico de biología, o en la manera de construirlo (ya sea a través de discusión oral, por resolución de problemas u otra). Centrar el intercambio en la forma de conocer un contenido requiere que el docente, a través de sus intervenciones, haga explícito, por un proceso de «metacomunicación» (Titone, 1981), el camino seguido -por el grupo clase-para comprender la secuencia de análisis del tema o actividad. De esta forma se revela la estrategia de construcción para dicho tema por un proceso de metaanálisis de las resignificaciones personales y grupales que surgieron en el diálogo.

Por último, la metodología usada por el docente heteroestructurante, autoestructurante o interestructurante (Not, 1983) también hará variar la relación comunicativa, adquiriendo docente y alumnos diferentes roles y su relación determinado grado de asimetría, participación y formas de indicar cuál es el conocimiento válido para esa clase. En los intercambios centrados en la heteroestructuración, el contenido estará organizado desde el exterior y es impuesto al alumno, que lo ignora, generando en éste un rol pasivo. En estos casos, llamado por algunos didactas método normativo o dogmático, el saber no se cuestiona y se presenta como acabado. En la autoestructuración, el alumno es el artesano de su construcción y el saber está ligado a las experiencias y necesidades de la vida. Didácticamente este método es llamado iniciativo y busca mejorar la motivación del alumno. Por último, el interestructurante es aquél en el que los factores determinantes de la construcción del conocimiento no están exclusivamente en el objeto, como en la primera, ni en el sujeto, como en la segunda, sino en la interacción entre ambos. Didácticamente este modelo se centra en la idea de que la construcción del conocimiento es responsabilidad del alumno, pero a partir de situaciones de enseñanza-aprendizaje organizadas por el docente y que pueden llevar al alumno a la autoestructuración.

Los aspectos teóricos anteriores conforman el marco desde el cual elaboramos la estructura de análisis para interpretar los diálogos, explicitado verbalmente, en clases de ciencias. Tomamos las intervenciones verbales como indicador de la forma en que emerge el conocimiento en el aula y le atribuimos un carácter dinámico e histórico, por ser un hecho que ocurre periódicamente entre las mismas personas, docente y alumnos, a lo largo de todas las clases de un año. Si bien describiremos sus componentes en un momento dado, consideramos que el sistema de comunicación evoluciona a lo largo del tiempo. Su forma más estable comienza cuando se comparten y amplían los códigos y contextos de referencia entre alumnos y docente y, en consecuencia, se afianzan las relaciones entre ellos, posibilitando que la participación del alumno crezca gradualmente, a medida que el docente cede parte de su control sobre el conocimiento y la forma de conocer.

\section{DESARROLLO EMPÍRICO}

La investigación, de la cual este estudio forma parte, es de carácter etnográfico y su metodología, interpretativa, con estudio de casos. Su propósito es elaborar un esquema de análisis que permita describir, interpretar y explicar las secuencias de diálogo que se generan en clases de ciencias, destinadas a enseñar determinado tema. Presentamos aquí la categorización e interpretación de las intervenciones de docente y alumno, como la primera etapa en búsqueda del análisis de la interacción.

Los casos corresponden a situaciones naturales de dos asignaturas de nivel medio de enseñanza, Biología (1r. año) y Elementos de física y química ( $2^{\circ}$ año), pertenecientes a una escuela provincial de gestión pública. A través de observación no participante, durante todas las clases de un año lectivo, se registran, en forma escrita y con audio, los diálogos entre los docentes y los alumnos.

Para el registro de dichos diálogos, desde el análisis teórico, se confeccionó una primera lista de categorías semiestructuradas, definidas operacionalmente a lo largo del estudio. Las mismas quedaron incluidas en cuatro niveles, que constituyeron un esquema, como protocolo de análisis de las intervenciones.

Nuestro propósito era que los niveles y categorías permitieran:

\section{- un análisis de tipo didáctico;}

- centrar la atención en el desarrollo y emergencia de los contenidos de cada clase;

- diferenciar el tipo de intervención del docente y del alumno respecto de su relación con el contenido;

- integrar la dimensión temporal para el análisis de la secuencia de construcción conceptual; 
- respetar el contexto de la tarea;

- caracterizar las clases y las formas en que se estructura el conocimiento a través del diálogo;

- identificar la estrategia particular que utiliza cada docente para construir y legitimar los diferentes temas con sus alumnos.

Las secuencias temáticas se reconstruyeron a partir del registro de las intervenciones verbales del docente y de los alumnos, en la totalidad de las clases sobre cada tema. El análisis fue de los temas siguientes: ecosistema (T1), factor abiótico-agua- (T2), factor abiótico-temperatura y calor- (T3) y energía (T4).

También se incorporó el contenido de sucesivas reuniones y entrevistas con los docentes, a fin de ir contrastando nuestras observaciones.

La elaboración de niveles y categorías se realizó a través de un proceso de teorización que culminó con el desarrollo de una tipología o clasificación de los modos de intervenciones de docente y alumnos. Las frecuencias se calcularon para completar la descripción. Para ello fue necesario enumerar los datos y codificar las intervenciones y los tiempos, separando el registro cada cinco minutos.

Luego de encontrar la estructura más acabada de nuestro esquema, se examinaron nuevamente dichos registros para buscar regularidades categoriales e intercategoriales. De esta forma surgieron las combinaciones que permitirían, más adelante, describir situaciones particulares de emergencia de determinado contenido en la clase. Actualmente estamos probando el proceso en nuevos casos y delimitando episodios.

En este artículo presentamos una primera parte del análisis correspondiente a la estructura del esquema y las frecuencias para cada categoría en cada uno de los temas.

\section{NIVELES DE ANÁLISIS DEL ESQUEMA}

\section{Nivel I. Contexto didáctico}

Es un nivel de análisis descriptivo e incluye todo aquello que particulariza la situación de enseñanza y de aprendizaje objeto de análisis. Pretende establecer los límites desde los cuales se realizará posteriormente la interpretación de las intervenciones.

- La primera categoría pertenece a la etapa preactiva de la clase.

En ella se registran datos de la institución, el curso, el docente y la planificación tanto de la materia para todo el año como de cada clase en particular. A partir de entrevistas con el docente y análisis de documentos (como planificación, cuaderno de clase, libro de aula), se identifica el tema o concepto a enseñar, su planificación, los recursos que deben utilizarse, el propósito y la ubicación en el currículo institucional del área y de la materia. También se explicita el nivel de conceptualización que el docente desea alcanzar y las actividades a partir de las cuales organizará su construcción. Del mismo modo se identifica cuál será el conocimiento nuevo para esa clase, ya sea de carácter conceptual, procedimental o actitudinal. Se registran los comentarios del docente sobre posibles dificultades que cree encontrar para la construcción de dicho conocimiento. Por último, se anota el tiempo estimado para su desarrollo y el tipo de clase que prevé dar el docente: si corresponde a la iniciación de un tema, a un desarrollo con mayor nivel de complejidad o a un repaso y cierre.

- La segunda categoría pertenece a la etapa activa.

Durante el desarrollo de las clases, se registran las intervenciones verbales de docente y alumnos y observaciones de todos los acontecimientos que pueden condicionar dichas intervenciones. Por ejemplo, imprevistos, interrupción de la clase, mal funcionamiento de algún recurso, indisciplina, clima del aula, infraestructura, entre otros. También se analiza la diferencia entre lo que se previó hacer y lo que se hace, principalmente las rupturas de contratos pedagógicos explícitos o implícitos.

- La tercera categoría pertenece a la etapa postactiva.

Luego de realizada la grabación de los diálogos y las observaciones en cada clase, se anotan las primeras interpretaciones, basadas en los registrados de las dos categorías anteriores. Además se recogen las opiniones del docente respecto de lo ocurrido en esa clase, principalmente las razones que éstos dan para los hechos no previstos en la planificación.

\section{Nivel II. Análisis de las intervenciones}

Es un análisis más detallado que identifica las diferentes alternativas de intervención, tanto del docente como del alumno. Incluye 23 categorías, designadas con letras desde la A hasta la $\mathrm{X}$ y divididas en interrogativas $\mathrm{y}$ afirmativas. Siguiendo el proceso descripto anteriormente para cada categoría y su aparición en el desarrollo de cada tema, se determina su frecuencias, distribución temporal, relación con otras categorías e interpretación didáctica.

En la tabla I se presentan las categorías del nivel II y sus frecuencias para los cuatro temas.

\section{Preguntas que formula el docente}

- A. Un primer grupo de preguntas es para controlar el conocimiento de los conceptos, procesos o términos previamente analizados y validados en clase o en otras disciplinas; es decir, lo que se sabe del tema hasta ese momento. Conllevan intención evaluativa respecto del 
Tabla I

Frecuencias (en porcentajes) de las categorías del nivel II referidas al tipo de intervención por tema.

\begin{tabular}{|c|c|c|c|c|c|}
\hline CATEGORÍAS & FINALIDAD & $\begin{array}{c}\text { TEMA } 1 \\
\text { Ecosistema }\end{array}$ & $\underset{\text { Agua }}{\text { TEMA 2 }}$ & \begin{tabular}{|c|} 
TEMA 3 \\
Calor y temperatura \\
\end{tabular} & $\begin{array}{l}\text { TEMA } 4 \\
\text { Energía }\end{array}$ \\
\hline & Preguntas del docente & & & & \\
\hline A & Control & 5,7 & 5,6 & 6,2 & 1,9 \\
\hline B & Indagar concepto propio & 8,2 & 14,9 & 12 & 9,2 \\
\hline $\mathrm{C}$ & Indagar comprensión de algo dado & 11,4 & 8,4 & 13,4 & 3,1 \\
\hline $\mathrm{D}$ & Sugerir respuesta & 0,6 & 0,8 & 2,1 & 0 \\
\hline $\mathrm{E}$ & $\begin{array}{l}\text { Provocar diversidad } \\
\text { de opinión }\end{array}$ & 5,1 & 0 & 0 & 0 \\
\hline \multirow[t]{2}{*}{ Subtotal } & & $\mathbf{3 1 , 0}$ & 29,7 & 33,7 & 14,2 \\
\hline & Afirmaciones del docente & & & & \\
\hline $\mathrm{F}$ & $\begin{array}{l}\text { Reubicar el aporte } \\
\text { del alumno }\end{array}$ & 1,3 & 6,8 & 4,5 & 3,1 \\
\hline $\mathrm{Ga}$ & Síntesis & 5,1 & 2,8 & 3,4 & 5,8 \\
\hline $\mathrm{Gb}$ & Información nueva & 3,8 & 2,8 & 5,1 & 4,2 \\
\hline Gc & Nombre & 3,2 & 0 & 0 & 0 \\
\hline Gd & Metaanálisis & 0 & 0,8 & 0,3 & 0,8 \\
\hline $\mathrm{H}$ & Aporte nuevo & 1,9 & 0,4 & 0 & 2,6 \\
\hline I & Consignar actividad & 5,7 & 6,4 & 3,4 & 15 \\
\hline $\mathbf{J}$ & Justificar tarea & 1,2 & 0,4 & 2,1 & 4,2 \\
\hline $\mathrm{K}$ & Regular participación & 3,8 & 3,2 & 3,8 & 5,4 \\
\hline $\mathbf{L}$ & Respuesta neutral & 0 & 0,4 & 0,7 & 0,7 \\
\hline $\mathbf{M}$ & Negación & 0 & 0,4 & 1 & 0 \\
\hline \multirow[t]{2}{*}{ Subtotal } & & 26 & 24,4 & 24,3 & 42 \\
\hline & Preguntas del alumno & & & & \\
\hline $\mathrm{N}$ & $\begin{array}{l}\text { Solicitar extensión } \\
\text { del tema }\end{array}$ & 0,5 & 0 & 0,3 & 0,8 \\
\hline $\mathrm{O}$ & Solicitar aclaración específica & 0,6 & 0 & 2,1 & 8,5 \\
\hline $\mathbf{P}$ & Confirmar una idea & 1,9 & 0 & 0,3 & 0,7 \\
\hline \multirow[t]{2}{*}{ Subtotal } & & 3 & 0 & 2,7 & 10 \\
\hline & Afirmaciones del alumno & & & & \\
\hline Q & Expresar conocimiento & 18,4 & 37,3 & 21,5 & 19,2 \\
\hline $\mathrm{R}$ & Dar opinión & 12,7 & 6 & 14 & 7,3 \\
\hline $\mathrm{S}$ & Repetir textualmente & 5,7 & 1,3 & 3,1 & 2,7 \\
\hline $\mathrm{T}$ & No contesta & 3,2 & 1,3 & 0,7 & 1,5 \\
\hline $\mathrm{U}$ & Solicitar pauta de trabajo & 0 & 0 & 0 & 0,4 \\
\hline $\mathrm{V}$ & $\begin{array}{l}\text { Solicitar pauta de } \\
\text { evaluación }\end{array}$ & 0 & 0 & $\mathrm{O}$ & 1,2 \\
\hline$x$ & Llamar la atención & 0 & 0 & 0 & 2,3 \\
\hline Subtotal & & 40 & 45,9 & 39,3 & 34 \\
\hline Total & & $100 \%$ & $100 \%$ & $100 \%$ & $100 \%$ \\
\hline
\end{tabular}


alumno y pretenden verificar su aprendizaje. Se repasa el concepto que se vio en clases anteriores. En la mayoría de los casos se busca explicitar el «término» que expresa dicho concepto, más que su explicación.

\section{Ejemplo tema 1}

1-D: ¿Qué vimos ayer? A

2-A: El método científico. (Q)

3-D: ¿Qué es? A

Suele revisarse, con igual finalidad, una actividad resuelta por los alumnos con el libro de texto buscando la correspondencia de la respuesta del alumno con lo expresado en dicho texto. Las contestaciones son «a coro», por tener un referente común:

\section{Ejemplo tema 1}

57-D: ¿Qué criterio tuvieron en cuenta para clasificar los ecosistemas? $\boldsymbol{A}$

58-A: Tamaño, profesora. ( $S$ )

59-D: ¿En qué los clasificaron? $\boldsymbol{A}$

60-A: Macro, micro y meso. $(S)$

Este tipo de preguntas no ocupó más del $6 \%$ de las intervenciones de las clases para los cuatro temas.

Su distribución temporal, a lo largo del desarrollo del tema, las ubica preferentemente en el comienzo de una clase retomando lo visto en la anterior. Forman parte de los acuerdos previos entre docente y alumnos, antes de una nuevo análisis.

$-B$. Un segundo grupo de preguntas es para indagar si el alumno comprende un concepto o proceso propio, expresado o experimentado por éste en intervenciones anteriores; ayuda al alumno a indagar su propia comprensión, ya sea para completar o modificar un concepto.

El docente suele parafrasear lo que dijo el alumno, incluyendo en su pregunta la respuesta dada anteriormente por él. Otras veces, se incluye alguna dificultad que provoca el replanteamiento de la respuesta del alumno.

El fin de este tipo de preguntas es estimular el pensamiento y la reflexión en la clase. No obstante, pudimos observar que no siempre que el docente replantea la respuesta de los alumnos es porque se suponga que es errónea, simplemente es para que siga con el razonamiento, confirme su idea o la explique mejor.

Por ejemplo, en el tema 2, al tratar el tema del agua (su relación con el suelo) y analizar lo que ocurre con la permeabilidad en diferentes suelos, registramos:
222-D: Se produce el ciclo del agua en el bosque? $\boldsymbol{B}$

223-A: Sí. (Q)

224-D: Pero pasamos y cortamos los árboles. ¿Qué pasa? $\boldsymbol{B}$

225-A: Las plantas no transpiran. (Q)

226-D: ¿Y qué pasa? B

227-A: No forman nubes. (Q)

228-D: ¿No o sí? Esperen... no se forman nubes porque en ese momento y en ese lugar no hay nubes. Pero puede ser que haya evaporación en otro lugar. $(F)$

En el tema 3, «Relación entre temperatura y calor», la docente plantea el análisis sobre la distribución de las plantas y la incidencia del Sol. A través del cuestionamiento a las escuetas respuestas de los alumnos, trata de que éstos retomen sus conocimiento cotidiano (basados en sus experiencias y observaciones) y brinden una explicación más completa y rigurosa del tema, que los aproxime al lenguaje y al contenido académico de la disciplina de biología.

250-D: ¿Considera que un vegetal aislado recibe más o menos luz que otros que están juntitos? $\boldsymbol{B}$

251-A: Más. (R)

252-D: ¿Por qué? $\boldsymbol{B}$

253-D: ¿Por qué vos consideras un vegetal aislado? $\boldsymbol{B}$

254-D: ¿Qué significa aislado? B

255-A: Solo. $(R)$

256-D: Recibe más o menos luz que otros que están juntitos. $\boldsymbol{B}$

257-D: Vamos... contesten. $(K)$

258-D: ¿Quién recibe más? $\boldsymbol{B}$

259-A: ¡Y! El aislado, porque tiene la ventaja de recibir luz más que los otros que están juntitos. $(R)$

260-D: ¿Por qué? B

261-A: Porque recibe luz por todas partes. $(R)$

262-D: ¿Le importa al tronco recibir luz? B

263-A: No. $(R)$

264-D: ¿Y en todo su follaje? B

Y así continua hasta la intervención 284.

Son uno de los tipos de interrogantes más frecuentes que plantean los docentes, especialmente en los casos en que la construcción conceptual parte de una idea del alumno 
y luego es fuertemente guiada por el docente. En general ocurren temporalmente alrededor de la mitad de la clase.

- C. Un tercer grupo es para indagar la comprensión de un concepto, experiencia o situación descripto en el enunciado de la misma pregunta del docente, en un libro de texto o en las validaciones previas (de conceptos o procesos) que realizó éste. El profesor los usa generalmente para provocar una plataforma común de conocimiento compartido. Intenta reflexionar sobre lo que el contenido significa para los alumnos y de alguna manera compararlo y acercarlo al significado que académicamente aceptará.

\section{Ejemplo tema 1}

8-D: Vamos a hablar de «macro», «micro» y mesoecosistema; es decir, grande, chico y mediano. Por ejemplo, «macro» es una selva, ¿y «micro»? $C$

45-D: Lo que tenemos a la izquierda es un ecosistema natural y los otros dos son ecosistemas humanos...(Señala una figura.) ¿Hay componentes del ecosistema natural que están en el ecosistema humano? $C$

Este tipo de interrogante C, como los anteriores B, son las más frecuentes en todos los temas. Es una manera de presentar el cuestionamiento al alumno, con una aclaración conceptual previa que cumple diferentes funciones: ubicarlo en el tema, validar una respuesta anterior, ofrecer la idea síntesis (o el término específico), presentar el contexto de la respuesta.

La diferencia con $\mathrm{B}$ es que en $\mathrm{C}$ el alumno no ha expresado previamente su conocimiento sobre el tema específico de la pregunta, sino que es el docente quien de alguna manera agrega una introducción que guía la posible respuesta.

En los casos analizados, se utilizó este tipo de intervención para acelerar el desarrollo de los temas. Por otro lado, podemos decir que son típicas de cuestionarios escritos. En las clases de física también se usaron en la presentación de situaciones problemáticas.

\section{Ejemplo tema 4}

$D$ : Un sistema líquido está a 15 grados centígrados y le ponemos un metal a 0 grados. ¿Qué pasará? $C$

- D. Un cuarto grupo de preguntas es para sugerir la respuesta a los alumnos, conformando el inicio de una frase a completar. El docente da «pistas» muy precisas a los estudiantes sobre el contenido que busca. Por ejemplo:

47-D: Estos componentes bióticos y abióticos forman parte de la...? $\boldsymbol{D}$

186-D: De los seres vivos, los que más influyen son...? D

Como puede observarse en la tabla I, son poco frecuentes en todos los temas y están ausentes en el último de ellos.
- E. Un quinto grupo de preguntas es para provocar diversidad de opiniones entre los alumnos. Generalmente no tiene que ver directamente con el tema y se hacen para generar otras ideas y abrir el debate sobre cosas conocidas por el alumno. Estimulan la participación y ocurren cuando el docente inicia una conversación colateral al tema de la clase. Es una manera de recuperar conocimientos que muchas veces, por procesos de diferenciación o analogía, pueden ayudar a comprender el nuevo contenido. Si bien las preguntas E pueden provocar análisis, éste no está organizado en el contexto del desarrollo del tema de la clase. Se producen circunstancialmente acorde con la participación e intereses del grupo. Aunque las intervenciones E estén ubicadas sucesivamente, no suelen tener hilo temático, se encadenan en función de las respuestas que el alumno va dando. En los casos observados sólo se usaron en el primer tema, cuando la docente retomó una charla de un indio mapuche dada para toda la escuela, recuperando algunos conceptos de éste al desarrollar el tema de ecosistemas humanos.

Por ejemplo 54-D: De todo lo que les contó (Se refiere al conferencista.), ¿qué aspectos mencionó de la vida de su pueblo? $\boldsymbol{E}$

\section{Aportes (afirmaciones) del docente}

- F. Son intervenciones afirmativas del docente que pretenden reubicar el aporte del alumno en el contexto del tema, de la disciplina o de lo que se pregunta sobre el tema. Estos casos ocurren generalmente luego de un malentendido y pueden estar referidos tanto a la forma de preguntar o al contenido de la pregunta del docente como al nivel de la respuesta del alumno. Es el ajuste que hace el enseñante para llegar al ámbito conceptual o terminológico de la respuesta que desea.

Estas intervenciones generalmente se hacen cambiando la entonación y retomando la respuesta del alumno.

\section{Ejemplo tema 1}

15-D: (Hablando de ecosistema natural y artificial... el docente expresa.) ¿Cuándo es artificial y cuándo natural? (A)

16-A: Cuando el hombre no interviene, es natural, y artificial cuando el hombre modifica la naturaleza o cuando el hombre lo hace. $(Q)$

17-D: Esos dos últimos son diferentes. Por ejemplo, yo les digo: «Chicos, haremos en el patio una laguna.» ¿Qué hice? $\boldsymbol{F}$

(En el mismo diálogo)

123-A: El agua sirve para erosionar el suelo. ( $Q)$

124-D: ¿El agua sirve para erosionar el suelo? $\boldsymbol{F}$

125-D: Comenzaremos de nuevo (El docente realiza un dibujo en el pizarrón y pregunta.) ¿Si acá traigo un trozo de suelo y con una regadera le tiro agua, ¿qué pasa? $\boldsymbol{F}$ 
El tema 4, por ejemplo, fue usado para señalar desde dónde buscar la respuesta:

38-D: ¿Saben ustedes por qué tipo de rayos se diferencian los colores en presencia de luz blanca? (A)

39-D: Investiguen. (I)

40-A: Puede ser porque el fenómeno es como un triangulito y salen los colores. $(Q)$

41-D: Eso es un prisma que descompone la luz en colores primarios. De allí, buscá la respuesta. $\boldsymbol{F}$

42-D: Busquen en los libros de física. $\boldsymbol{F}$

Estas intervenciones son reguladores de la construcción conceptual que se da en el aula. El grado de estructuración de las secuencias que surgen depende del contenido de la pregunta y de la frecuencia en que ocurran.

En los casos observados fueron más usados en el segundo y tercer tema, en momentos en que los alumnos no lograban contestar lo que el docente esperaba.

También, en temas como el de la energía, que resultan complejos al alumno, ya que muchas veces están alejados de su experiencia, el docente debió -con más frecuencia que en otros casos- recontextuar las respuestas para retroceder en el análisis del tema hasta encontrar el referente que ellos poseían, de lo contrario no podía continuar con el intercambio.

- G. Expresan aportes que indican la respuesta válida a través de:

a) la síntesis de los aportes de los alumnos o también aceptando la respuesta de uno de ellos en especial;

b) información nueva dando la definición o la explicación de un nuevo concepto o completando la idea que se estaba desarrollando en clase;

c) el nombre del concepto elaborado en clase;

d) el proceso seguido para elaborar el concepto; revisar el «cómo se conoce», por algunos autores llamado metacomunicación ${ }^{2}$ y considerado como lo propio de la enseñanza.

El objetivo es definir y expresar verbalmente cuál es el contenido más correcto, ya sea conceptual, procedimental o actitudinal. Es el que el alumno registra con más frecuencia en su cuaderno y el que luego se retoma en las evaluaciones.

\section{Ejemplos tema 1}

32-D: Por lo tanto, el ecosistema humano está constituido por componentes naturales y humanos. Muy bien, ¡correcto! $\boldsymbol{G a}$

$D$ : Biotopo más biosenosis es igual a ecosistema. $\boldsymbol{G} \boldsymbol{b}$

140-D: Estos que mencionamos se llaman componentes del ecosistema humano. $\boldsymbol{G} \boldsymbol{c}$

\section{Ejemplo tema 4}

244-D: Hay algunas intuiciones, pero lo fundamental es que ustedes sepan que ya hemos definido los conceptos aunque no se den cuenta. $\boldsymbol{G d}$

Los casos observados son el tipo de intervención afirmativa del docente más frecuente y, en general, se presentan en la segunda mitad de la clase, con la finalidad de cerrar el desarrollo del tema y legitimar los conceptos y procesos que surgieron.

- H. El docente realiza un aporte para contribuir con un elemento conceptual adicional, como si fuera un alumno. Generalmente son los propios pero a veces el enseñante simula y relata ejemplos que dieron otros alumnos. Algunas veces hace referencia a intervenciones anteriores.

\section{Ejemplo tema 4}

205-D: Ya que ustedes dicen su definición, yo voy a decir la mía y me ayudan a ver qué aspecto toco... (A continuación el docente definió energía.) $\boldsymbol{H}$

Es un tipo de intervención muy poco frecuente que ubica al docente en un rol diferente al habitual y a la cual el alumno no está acostumbrado. En el caso del desarrollo del tema 4, fue un estrategia planificada por el docente en el marco de un enfoque constructivista para la enseñanza de la física.

- I. El último tipo de intervención afirmativa es para consignar una tarea, un tipo de análisis o una secuencia conceptual a seguir. A veces se refiere a un criterio de evaluación.

\section{Ejemplo tema 3}

17-D: El resultado que les ha dado, ahora lo veremos en autores científicos, para ello revisaremos el tema en el libro de texto y cotejaremos nuestros datos. I

\section{Otras intervenciones del docente}

- J. Estas intervenciones sirven para indicar o justificar el tipo de tarea independientemente del contenido; por ejemplo, decirles que trabajar en grupos pequeños de dos o tres alumnos es mejor que en grupos de cinco.

- K. Para estimular, controlar e indagar el seguimiento, atención o participación. Por ejemplo, animarlos con una broma o interrumpir la clase y preguntar a alguien que está distraído.

- L. Para contestar algo neutral y dar pie a que siga la conversación sobre el tema entre los alumnos.

- M. Para negar un aporte del alumno.

En general, como puede observarse, en la tabla I, las intervenciones del docente (tanto interrogativas como 
afirmativas) cubren poco más del $50 \%$ de lo que dura el tratamiento de cada tema. En los tres primeros, a diferencia del cuarto, el docente interroga más veces de las que afirma. En el cuarto tema es notable la mayor frecuencia de afirmaciones del docente como de diversidad de intervenciones.

\section{Preguntas que formulan los alumnos}

- N. Para solicitar extensión del tema, buscando más información. Surgen generalmente cuando las consignas no están claras por su nivel o tipo de formulación.

\section{Ejemplo tema 3}

248-A: ¿Cuándo hay temperatura baja y los tubos capilares se rompen, ¿qué pasa con las plantas?, ¿cómo se vuelven a recomponer? $N$

- O. Para solicitar una aclaración específica referida a un ejemplo dado en clase, un término, un concepto o una respuesta posible. En este caso los alumnos buscan identificar el contexto válido para la respuesta que quieren expresar o la transferencia a algo que conoce. Ocurre tanto para los contenidos conceptuales, procedimentales como para los actitudinales.

\section{Ejemplo tema 1}

23-A: ¡Profesora! ¿La plaza España es un ecosistema artificial? $\boldsymbol{O}$

- P. Para confirmar una idea propia o de otro (compañero o docente). Esto lo hace generalmente repitiendo su respuesta o apoyando a un compañero. Surge como paso posterior al tipo $\mathrm{O}$ de pregunta y representa la explicitación de la propia construcción conceptual.

\section{Ejemplo tema 1}

Ante la afirmación de la docente de que el ecosistema es vida e implica actividad, el alumno pregunta:

84-A: ¿Si las palomas están quietas? $(O)$

85-D: Lo mismo hay actividad. (Gb)

86- $A$ : Pero, si estamos en un museo, hay relaciones porque estamos nosotros. ¿No es así? $\boldsymbol{P}$

Las intervenciones interrogativas del alumno, en general, cubren un porcentaje muy bajo de los diálogos, representando menos del $5 \%$ del total de las intervenciones para dicho tema, excepto en el tema 4 (energía) en el que la docente desarrolla situaciones de construcción conceptual compartidas con los alumnos, y las preguntas de tipo O llegaron a un $8 \%$ de las intervenciones.

\section{Aportes que hacen los alumnos}

- Q. Para expresar su conocimiento o interpretación sobre un concepto de la disciplina o proceso de com- prensión conceptual por solicitud del docente. Éste los utiliza para detectar el grado de elaboración conceptual; por ejemplo, si existen preconceptos o algún tipo de relación conceptual que dificulte o no el desarrollo posterior. Constituyen las intervenciones más frecuentes de los alunmos, por ser el tipo de aporte que más espera el docente que éstos hagan. En los análisis de secuencia temporal que hicimos, para cada tema, aparecen tiempos prolongados con presencia de Q. En algunas etapas de los diferentes temas, indican que se está realizando un trabajo grupal (en grupos pequeños en los que todos participan) o son respuestas consecutivas de varios alumnos.

Podemos decir que son este tipo de intervenciones las que definieron la participación del alumno en las clases observadas, principalmente en los temas 2 y 3 .

Generalmente el alumno presenta aquí una argumentación válida en el contexto del desarrollo de ese tema, es decir, con el nivel de formalidad esperado.

- R. Para dar su opinión sobre un aporte o experiencia propia o de otro compañero. Puede consistir en ejemplos pero que, a diferencia de $\mathbf{Q}$, están fuera del contexto de la disciplina. Tienen que ver con expresiones, generalmente en términos de lenguaje cotidiano. A veces surgen de transferencias o comparaciones que hacen los alumnos sobre el tema de la clase, en un contexto diferente.

Estas contribuciones de los alumnos se presentan en climas de aula donde hay confianza con el docente. Son útiles para evidenciar preconceptos. Si bien no constituyen directamente temas de evaluación para el docente, éste puede sacar a la luz analogías que hace el alumno y que posteriormente se pueden retomar.

Es problemático para el investigador categorizar este tipo de intervención, ya que depende del nivel de formalidad del desarrollo del tema en cada clase, por cada grupo y cada docente. Generalmente nos apoyábamos por las validaciones $(\mathrm{G})$ o contextuación $(\mathrm{F})$ del docente que interviene y que ocurrían a continuación de intervenciones $\mathbf{R}$ del alumno.

\section{Ejemplo tema 3}

48-D: La temperatura puede ser alta o baja.¿Qué significa que la temperatura sea alta o baja? $(C)$

49-A: Si es alta, yo tengo calor, hace calor. $\boldsymbol{R}$

50-D: Él dice que temperatura alta significa más calor y baja, menos calor. ( $G a)$

D: Entonces, ¿qué nos dice calor y qué, temperatura? (C)

- S. Para repetir textualmente en referencia a una respuesta ya validada, sobre algo que dice el libro o que dijo la profesora anteriormente. No hay elaboración por parte del alumno. 
Es un caso típico de aparición frecuente de $\mathrm{S}$ cuando el docente utiliza cuestionarios muy estructurados que conducen al alumno a responderlo sólo con lo expresado en el libro de texto y de la misma manera. Forman parte de lo que llamábamos metodologías heteroestructurantes.

En los casos observados son poco frecuentes y están fuertemente relacionadas con la aparición de preguntas tipo A.

\section{Otras intervenciones del alumno}

- T. Hemos registrado como un tipo de intervención el hecho de que el alumno no conteste, ya que los silencios son indicadores de malentendidos. Por ejemplo, cuando los alumnos han respondido varias veces, el docente insiste con la misma pregunta y ellos no entienden el contexto de la pregunta; también cuando desconoce el lenguaje o cuando no sabe qué opinar.

- U. Para solicitar pautas de trabajo independientemente del tema de que se trate.

- V. Para solicitar pautas de evaluación. Generalmente se originan cuando no queda claro al alumno cuál es el conocimiento válido para el docente.

- X. Para llamar la atención. Por ejemplo, preguntando sobre otro tema, preguntando de nuevo a pesar de haber entendido, poniendo alguna condición al docente.

Como se puede observar en la tabla I, las intervenciones verbales de los alumnos corresponden al $40 \%$ del tiempo aproximadamente, de éstas la mayoría son afirmativas. Sólo vimos un número significativo de preguntas del alumno en dinámicas de clase generadas para tal fin, como fue la del tema 4.

\section{Nivel III. Inferencias didácticas}

En este nivel planteamos hipótesis que surgen de las relaciones entre las diversas categorías de los niveles anteriores, de la interpretación de los datos y de las secuencias de aparición de las intervenciones en el desarrollo de cada tema.

Son inferencias didácticas porque intentan explicar lo que sucedería con la situación de enseñanza-aprendizaje y con la comprensión de un determinado conocimiento, en un contexto de comunicación con un grado de asimetría particular.

Este nivel de análisis utiliza como fuente las categorías de los dos niveles anteriores y es quizás el que más ayuda a una reflexión didáctica. Sólo se expresa en forma de hipótesis, ya que demostrarla implicaría comenzar con otras investigaciones y proponerla como solución se convertiría en una normativa. Por otro lado, las causas de las situaciones que aparecen no derivan solamente de la forma en que circula el conocimiento en el aula. La misma complejidad y singularidad de este tipo de situaciones poseen otros elementos que la condicionarían.
Mencionaremos a continuación algunos ejemplos de dichas hipótesis, que acompañan la interpretación didáctica que se hizo para cada tema.

\section{Ejemplos}

- Si se usa de forma reiterada $\boldsymbol{D}$ (sugerir respuesta), en desmedro de otras alternativas, surgen clases con escasa participación del alumno y generalmente, cuando la hay, se limita a la aceptación sin cuestionamiento de los aportes del docente $(\boldsymbol{S})$.

- Favorecer $\boldsymbol{B}$ (indagar conceptos propios) contribuye al proceso de reflexión en el alumno sobre su propia comprensión; no obstante, su uso reiterado puede conducir a procesos netamente inductivos si es muy guiado por el docente.

- La frecuente necesidad de un docente de reubicar lo que el alumno responde a su pregunta, a través de $(\boldsymbol{F})$, indica, además de una negociación del aporte, una ausencia de códigos comunes en la comunicación, un problema en la manera de establecerlos o falta de conocimientos previos para comprender lo que se pregunta.

- La aparición de las intervenciones $\boldsymbol{F}$ o $\boldsymbol{B}$ después de un aporte del alumno no siempre son necesarias para continuar la construcción conceptual.

- La aparición frecuente de $\boldsymbol{G} \boldsymbol{b}$ (información nueva) disminuye la participación del alumno en la construcción del conocimiento e indica, por ejemplo, que el docente desea ahorrar tiempo en el tratamiento de ese tema, imponiendo a la clase un ritmo, a veces diferente del que los alumnos pueden seguir.

- La presencia de $\boldsymbol{G} \boldsymbol{d}$ (metaanálisis) en una secuencia de diálogo, especialmente al final, indica que el docente se interesa no sólo por el conocimiento sino también por la «forma de conocer», planteando lo que los psicólogos llaman «sesiones de razonamiento público»; es de esperar este tipo de intervenciones si la propuesta didáctica es de tipo constructivista.

- El texto de las respuestas $\boldsymbol{G}$ (validaciones conceptuales) del docente tiene un nivel de complejidad diferente al del alumno ( $\boldsymbol{Q}$-conocimiento) y al del libro de texto, relacionado con el conocimiento enseñado y la lógica de la interacción.

- Cuando el docente requiere frecuentemente $\boldsymbol{R}$ (respuestas de opinión), indica que está interesado por construir el conocimiento a partir de lo que el alumno ya sabe a partir del conocimiento cotidiano. Por ello es más frecuente $(\boldsymbol{R})$ en el comienzo del tratamiento de un tema o cuando algo no se entiende y se debe volver a explicar.

- El uso reiterado de $\boldsymbol{V}$ (solicitar pautas de evaluación) por parte del alumno evidencia su necesidad de aclarar las pautas del «contrato» de aprobación de la materia, a veces independiente del contenido. Esto mostraría lo que muchos autores llaman el «aprender cómo navegar en el aula» más que un conocimiento. Generalmente su 
aparición ocurre en clases donde al alumno no le queda claro el propósito del docente y no logra involucrarse en la secuencia que éste establece.

- A medida que se requiere mayor nivel de abstracción y formulación, el docente interroga menos, ya que debe dedicar más tiempo a incluir conceptos nuevos, recontextualizar las respuestas del alumno o a expresar la respuesta válida.

- A medida que avanza el año escolar y mejora la comunicación docente-alumno, los docentes disminuyen las intervenciones con intención evaluativa y generan situaciones que permiten la aparición de interrogantes del alumno.

\section{Nivel IV. Síntesis conceptual}

Este nivel de análisis es síntesis de todos los anteriores y acota la interpretación a lo que sucede con el contenido en cuestión. Aquí se extraen las relaciones conceptuales que se van elaborando a lo largo del diálogo y los procesos (generalmente guiados por el docente) que generan dichas relaciones.

A partir de este análisis podemos decir que:

- se mantiene el eje conceptual, definido por el docente como conocimiento a enseñar, a lo largo de toda la clase;

- lo que ocupa su lugar es otro concepto o nivel de conceptualización;

- es un aspecto semántico o sintáctico del contenido lo que guía la construcción;

- se establece una secuencia continua y lógica de análisis del tema;

- quedan aspectos del tema sin cerrar o validar;

- el docente vuelve al contexto compartido para reiniciar el tratamiento del tema o si lo impone sin contemplarlo.

Este análisis requiere la subdivisión por clases y por momentos de la clase (apertura, desarrollo y cierre), comparando los diagramas conceptuales de los diferentes momentos.

Aislar la secuencia que se genera con el tipo de intervención $\boldsymbol{G}$ (validación conceptual) permite visualizar el esqueleto de la lógica del desarrollo del tema.

El resultado de este nivel son diagramas conceptuales para cada tema. Su análisis didáctico permite ver la distancia entre la lógica del contenido a enseñar y la lógica de la interacción provocada por la enseñanza.

\section{DISCUSIÓN}

Interpretar desde la didáctica de las ciencias las intervenciones de docentes y alumnos durante el desarrollo de la clase es una tarea compleja tanto desde su perspectiva teórica como de la empírica.

Si bien sabemos que no todas las clases de ciencias privilegian las intervenciones de tipo verbal, en las escuelas de nuestro medio y a nivel secundario, son el modo más usado por el docente para tratar un tema de esta disciplina, principalmente cuando los estudiantes plantean sus dudas.

Nuestro interés fue indagar situaciones de negociación y construcción de conocimiento manifestadas en el diálogo verbal docente-alumnos.

Para la mayoría de los temas de investigación en didáctica de las ciencias, la principal base de datos y referentes están en el aula y en los procesos de comunicación que en ella ocurren; basta sólo pensar en el análisis de las preconcepciones, errores conceptuales, modelos de participación, cambio conceptual, clima del aula, etc.

A través de las interpretaciones de los diálogos pudimos aproximarnos a la comprensión de los procesos de transacción que ocurrían en la clase. Para ello comenzamos por considerar la teoría didáctica como fuente de comprensión y reflexión sobre la práctica más que como previa a la misma y generadora de prescripciones. No buscamos generalizaciones que delimitaran un modelo de instrucción, más bien pretendimos identificar los códigos y normas que regulan la singular relación comunicativa de un contenido enseñado, es decir, comprender «cómo funciona» el intercambio. Detectamos la necesidad de un conocimiento tipológico explicativo del proceso de diálogo, tendiente a reconstruir el escenario observado.

Para la interpretación didáctica importa describir la situación de asimetría en su totalidad, tanto su estructura como su secuencia y evolución. El esquema que presentamos en este artículo es la base desde la cual hacemos dichas interpretaciones.

Cada clase conformó una estructura singular y se dibujó en ella una trama de relaciones que evolucionó a medida que sus agentes (docente y alumnos) compartían más tiempo y se comunicaban desde el mismo contexto disciplinar. Lo anterior nos llevó a confirmar que un esquema con categorías semiestructuradas sería más útil para interpretar y captar el sentido de dichas situaciones.

Los cuatro niveles del esquema resultaron interdependientes y con diferente grado de profundidad. El primero permitió describir el contexto de las clases en sus tres momentos. El segundo, más analítico, se organizó en categorías de preguntas y respuestas. Las mismas ayudaron a encontrar el objetivo para cada intervención. En la etapa siguiente de la investigación (no incluida en este artículo) se agregó, a la clasificación, un análisis de las secuencias, de las combinaciones categoriales más usadas y se identificaron los episodios en que éstas ocurrían. Así, se pudieron delimitar situaciones específicas, por ejemplo, de refutación, contextuación, evaluación, etc., en cada tema, para cada docente y en diferentes momen- 
tos de la clase. Los datos de la segunda etapa completaron la interpretación didáctica, sistémica y reflexiva, que esperábamos.

El tercer nivel ayudó a predecir lo que ocurría con el proceso, si predominaba algún tipo de intervención o combinación secuencial de ellas. Brindó al diseño de análisis una estructura más abierta que promovió la reflexión hacia nuevas líneas de investigación. También a partir de aquí se pudo seguir reajustando los otros niveles del esquema. Consideramos de fundamental importancia, para la didáctica, el planteo de la reflexión de lo que sucede en la clase en términos de hipótesis.

Por último, el cuarto nivel saca a la luz la verdadera secuencia de contenidos reconstruida en la presentación de una clase, cuánto se aleja o acerca de lo previsto la lógica elaborada a partir del conocimiento enseñado.

Como dijimos al comienzo del artículo, se presenta aquí sólo una parte de la investigación, ya que falta el análisis sobre el eje temporal, es decir, cómo se sucedieron los diferentes tipos de intervenciones y qué situaciones de interacción se generaron. No obstante, a partir de las frecuencias presentadas en la tabla I, podemos extraer conclusiones preliminares de la estructura de la intervención en los cuatro temas.

Los tres primeros temas -ecosistema, agua, calor y temperatura (T1, T2 y T3)- fueron desarrollados por una docente de biología y el cuarto -energía (T4)-, por una profesora de física. Comparando los temas podemos realizar las siguientes reflexiones:

- Las clases de biología (T1, T2 y T3) se caracterizaron por la dinámica de sus diálogos estimulados por los análisis de la docente sobre las intervenciones de sus alumnos, indagando permanentemente sus comprensiones y conocimientos. Lo anterior se manifiesta en el porcentaje elevado de preguntas del docente, cubriendo el $30 \%$ del total de las intervenciones para cualquiera de los tres temas que desarrolló. Así, la estrategia más usada fue una exposición dialogada y un «diálogo guiado» a través de la inducción de conceptos. En estas clases emergen dos tipos de conocimientos: los referidos a las relaciones entre conceptos ya vistos y discusiones sobre los términos que expresan dichos conceptos, ambos indicados en el mayor porcentaje de intervenciones tipo $\boldsymbol{F}$, especialmente en los temas 2 y 3 .

- Los aportes de los alumnos generalmente fueron de conocimiento $(Q)$ y pocos de opiniones espontáneas $(R)$. La participación de éstos se generó por el desafío que provocaba el cuestionamiento permanente de sus respuestas, estando ausentes sus propios interrogantes $(O)$.

- Las clases de física (T4) correspondían a la planificación de una propuesta constructivista. Pudimos observar que se volcó la responsabilidad de la construcción del conocimiento al grupo de alumnos y éstos debieron trabajar haciendo uso de sus propios conceptos. La estrategia más empleada por la docente fue la secuencia de actividades que indicaban el análisis conceptual -categorizada en nuestro esquema como $I-$ y que, en este caso, cubrió un $15 \%$ de las intervenciones. Las validaciones $(G)$ se hicieron en referencia a estas secuencias y también a conceptos, pero en menor porcentaje que la docente anterior.

La profesora de física, más que preguntas, planteaba tareas, y el alumno las realizaba, exponía sus dudas y expresaba sus opiniones. Se conformaron clases con características diferentes a la de biología, ya que aquí se observa variedad en las intervenciones de los alumnos y un porcentaje importante de interrogantes de ellos $(\boldsymbol{O})$, además de las clásicas respuestas de opinión $(R)$ y conocimiento $(Q)$. También aparecen en este tema otras categorías como $U, V$ y $X$; no obstante, el porcentaje total de intervenciones del alumno es inferior al caso anterior. Esto se debe a que la profesora debió dedicar mucho tiempo en explicar cuál era la dinámica de la clase $(J)$ y a regular la participación $(K)$, por lo novedoso de la metodología.

- Los conocimientos válidos $(G)$, en ambos casos, emergieron más frecuentemente de la síntesis de los aportes de los alumnos al diálogo didáctico durante el desarrollo del tema $(G a)$. En ambos, también, sólo el $4 \%$ de las intervenciones docentes fue para introducir conceptos nuevos $(G b)$.

- Debido al predominio de preguntas que inducen los conceptos en las clases de biología , éstas se evidenciaron como más estructuradas y planificadas. Lo anterior se manifiesta en el predominio de preguntas que inducen los conceptos; podríamos decir que se enfatizan los aspectos «semánticos» de la disciplina (conceptos y relaciones conceptuales en biología). En física se planifica la metodología y las actividades de los alumnos, pero no se define una lógica particular para la interacción con las respuestas de éstos y la construcción del tema. Aquí el predominio se ubica en los aspectos «sintácticos» (procedimientos de razonamiento teórico en física).

Si bien no podemos en el artículo presentar todo el diálogo para cada tema, adelantaremos breves conclusiones sobre ellos:

- Sólo en las clases del tema 1 «Ecosistema» se manifiesta una intención evaluativa por parte de la docente debido al planteamiento de preguntas de conocimiento $(A)$ y de respuestas textuales $(S)$. El principal referente de validez conceptual fue el libro, a diferencia del resto de los temas.

- Lo particular del tema 2 «Factor abiótico agua» fue su finalidad. La frecuente aparición de F-preguntas de recontextuación- y el contenido de las mismas permite decir que la intención de la docente era que el alumno comprendiera «el tipo de análisis» más que los conceptos.

- En el tema 3 se planteó un análisis conceptual diferente al resto. A partir de un cuestionario escrito se buscaba un conjunto de respuestas que integraran y relacionaran 
diferentes conceptos. Se necesitó la bibliografía pero también saber interpretarla y usarla, ya que las respuestas no estaban expresadas directamente en los textos. Para analizar la influencia del calor y la temperatura sobre el crecimiento de las plantas fue necesario entender primero algunos fenómenos físicos. Este hecho condujo a un intercambio verbal entre docente y alumnos, que requirió preguntas del docente sobre conceptos que él introducía $(C) \mathrm{y}$, consecuentemente, respuestas de conocimiento por parte de los alumnos $(C)$.

- El tema 4 temporalmente abarcó la suma de los tiempos de los otros tres, por la metodología elegida.

El docente, como regulador del diálogo, actúa estructurando la construcción del conocimiento, de una determinada forma. La misma puede ir desde plantear una fuerte asimetría de roles hasta generar una autoestructuración en el alumno. Es justamente esta asimetría, el grado de participación del alumno y la manera de retomar y validar los conocimientos lo que diferencia una situación de otra y lo que debe analizar la didáctica de las ciencias.

El discurso es parte del proceso educativo, tiene una contextualización situacional pero, a su vez, genera su propio contexto en la medida que se va elaborando en su presentación. La interacción que provoca hace circular los mensajes y enfrenta argumentaciones que derivan de los significados personales y sociales.
El esquema presentado ayuda a elaborar el registro de base para un análisis didáctico de dicho discurso. Centran su atención en el desarrollo y emergencia de los contenidos de cada clase e identifican diferentes intervenciones, respetando el contexto de la tarea.

Nos queda por comentar su utilidad para diferenciar clases, identificar la estrategia particular que utiliza cada docente con el fin de construir y legitimar los diferentes temas y situaciones de construcción conceptual, a través de la integración de la dimensión temporal en el análisis, como así también ver las posibilidades que brinda para diseñar situaciones prototípicas de construcción conceptual.

\section{NOTAS}

${ }^{1}$ Acorde con las entrevistas realizadas con los docentes observados se considera «respuesta válida» aquélla que coincide con lo que se expresa del tema en textos especializados, con lo que estaba previsto que ellos enseñaran y los alumnos aprendieran o con su propio conocimiento sobre el tema. Esto último ocurre especialmente en docentes con muchos años de experiencia.

${ }^{2}$ Se habla de metacomunicación, según Titone (1986, p. 39), «cuando los interlocutores se plantean de manera directa y expresa [hablan de] cómo se desarrolla su relación-se comunican sobre cómo se comunican-. De esta manera se hacen explícitos los patrones relacionales [...] y con frecuencia es la única manera de resolver la expectativa contradictoriaen los participantes.

\section{REFERENCIAS BIBLIOGRÁFICAS}

ASTOLFI, J.P. y DEVELAY, M. (1989). La Didactique des Sciences. Presses Universitaires de France.

BARNES, D., BRITTON, J. y ROSEN, H. (1969). Language, the Learner and the School. Harmondsworth: Penguin.

BERTALANFFY, L. (1976). Teoría general de los sistemas. México: Fondo de Cultura Económica.

BRUNER, J. (1988). Desarrollo cognitivo y educación. Madrid: Morata.

BELLACK, A. et al. (1966). The language of the classroom. Nueva York: Teachers College, Columbia University. (Citado por Titone, 1981)
BERNSTEIN, B. (1977). Class, codes and control. Londres: Routledge and Kegan Paul Ltd. Trad. cast. (1989). Clases, códigos y control. I y II. Madrid: Akal, SA.

BERNSTEIN, B. (1988). Poder, educación y conciencia social de la transmisión cultural. Santiago: CIDE.

BROUSSEAU, G. (1996). Utilidad práctica de los enfoques teóricos para la organización y conducción de las actividades didácticas. Curso desarrollado en FAMAF-UNC,12 al 22 de agosto.

CANDELA, M.A. (1991). Argumentación y conocimiento científico escolar. Infancia y aprendizaje, 55, pp. 13-28. 
CAZDEN, C.B. (1988). Classroom Discourse. The language of teaching. Portsmouth: Heineman Educational Books. Trad. cast. (1991). El discurso en el aula. El lenguaje de la enseñanza y el aprendizaje. Barcelona: Paidós.

DE LONGHI, A.L. (1994). Alternativas de investigación en didáctica de las ciencias. Revista de la Universidad Blas Pascal, 2(5), pp. 1-9.

DE LONGHI, A.L. (1995). La construcción del conocimiento en el aula: un esquema y proceso de análisis. Tesis doctoral. Universidad Católica de Córdoba.

DOYLE, W. (1977). Learning the classroom environment: an ecological analysis. Journal of Teacher Education, 28(6), pp. 51-55.

DRIVER, R. (1989). «Students» conceptions and the learning of science. International Journal of Science Education, 11(5), pp. 481-190.

EDWARDS, D. y MERCER, H. (1987). Common Knowledge. Londres: Methuen/Routledge Trad. cast. (1988). El conocimiento compartido: El desarrollo de la comprensión en el aula. Barcelona: Paidós-MEC.

FENTERMACHER, G. (1986). Tres aspectos de la filosofía de la investigación sobre la enseñanza, en Wittrock. $L a$ investigación de la enseñanza I (2a. ed.). México: Fondo de Cultura Económica.

KLAASSEN, C.W. y LIJNSE, P.L. (1996). Interpreting Students'and Teachers' Discourse in Science Classes: An underestimated problem? Journal of Research in Science Teaching, 33(2), pp. 115-134

LLORENS, J.A. (1987). El uso de la terminología científica en los alumnos que comienzan el estudio de la química en la enseñanza media. Una propuesta metodológica para su análisis. Enseñanza de las Ciencias, 5(1), pp. 33-40.

LLORENS, J.A. et al. (1989). La función del lenguaje en el enfoque constructivista del aprendizaje de las ciencias. Enseñanza de las Ciencias, 5(1), pp. 33-40.

MAYOR SÁNCHEZ, J. (1983). Interacción, comunicación y lenguaje. Revista de Psicología General y Aplicada, 38(2), pp. 251-295.
MORTIMER E.F. (1996). Elaboração conceitual e lenguagem na sala de aula de Química e Ciências, Memorias de III Escuela Latinoamericana sobre Pesquisa em Ensino de Física, Canela R S, 1 al 12 de julio.

NOT, L. (1983). Las pedagogías del conocimiento. (2a. ed.). México: Fondo de Cultura Económica.

PERRET-CLERMONT, A. (1984). La construcción de la inteligencia en la interacción social. Madrid: Visor.

PÉREZGÓMEZ, A. (1985). La comunicación didáctica. Málaga: Universidad de Málaga.

PLEYAN, C. y NOGALES, V. (1990). Lenguaje y pensamiento en la trasmición del conocimiento científico. Cuadernos de Pedagogía, 67-68, pp. 13-15.

SEEGER, F. (1991). Interaction and knowledge in mathematics education. Recherches en Didactique des Mathematiques, Ed. La Pensée Sauvage, 11(2.3), pp. 125-166.

SINCLAIR, J.M.H. y COULTHARD, R.M. (1975). Towards an Analysis of Discourse: The English used by Teachers and Pupils. Londres: Oxford University Press. (Citado por Stubbs, 1987.)

STUBBS, M.(1984). Language, Schooland Classrooms. Londres: Methuen and Co. Ltd. Trad. cast. (1984). Lenguaje ye scuela: análisis sociolinguístico de la enseñanza. Buenos Aires: Cincel-Kapeluz.

THORRLEY, N.R. y STOFFLETT, R.T. (1996). Representation of Conceptual Change Model in Science Teacher Education. Science Education, 80(3), pp. 317-339.

TITONE, R. (1981). Il linguaggio nella interazione dedattica. Roma: Bulzoni. (Trad. cast. El lenguaje en la interacción didáctica: teorías y modelos de análisis. Madrid: Narcea).

VYGOTSKY, L.S. (1979). El desarrollo de los procesos psicológicos superiores. Barcelona: Crítica.

WATZALAWICH,P.(1981).Teoría de la comunicaciónhumana. Barcelona: Herder.

[Artículo recibido en abril de 1998 y aceptado en noviembre de 1999.] 